\title{
A Two-Bit Reflectarray Element Using Cut-Ring Patch Coupled to Delay Lines
}

\author{
Binh Duong Nguyen, Van-Su Tran, Linh Mai, Phuc Dinh-Hoang \\ School of Electrical Engineering, International University, Ho Chi Minh City, Vietnam
}

Correspondence: Binh Duong Nguyen, nbduong@hcmiu.edu.vn

Communication: received 10 May 2015, revised 3 January 2016, accepted 9 January 2016

Online publication: 9 March 2016, Digital Object Identifier: 10.21553/rev-jec.97

The associate editor coordinating the review of this article and recommending it for publication was Dr. Le Vu Ha.

\begin{abstract}
In this paper, a two-bit element for reflectarray applications is presented. The proposed element is based on the cut-ring patch coupled to delay lines through an annular slot. The four states of the reflection phase with a step of $90^{\circ}$ are achieved with two switches. This reported work is the first step towards the 2-bit active reflectarray element. Prototypes of the element have been fabricated and characterized in X-band using waveguide simulator. Measured results show good characteristics with low magnitude losses and the bandwidth of 1.7 -bit resolution is over $5 \%$.
\end{abstract}

Keywords- reflectarray element, reflectarray antenna, multilayer.

\section{INTRODUCTION}

Over the last two decades, microstrip reflectarray antennas have received considerable interests in radar, satellite applications due to their low cost, light weight, high efficiency. The reflectarray antennas combine some of the best features of conventional reflector antennas and array antennas. Different to the parabolic reflector antenna, microstrip reflectarray antennas are flat. The reflectarray antennas consist of a planar array of microstrip patches illuminated by a primary source. Each reflectarray element is designed to re-radiate the incident wave and to compensate for the spatial phase delay caused by the different path lengths from the illuminating feed to each element, so that the energy focuses on a specific direction.

Currently, there are great interests in electronically reconfigurable microstrip reflectarray antennas due to its advantages over mechanically steering antennas for the purpose of vibration reduction and maintenance. In the design of electronically reconfigurable reflectarray antennas, the critical point is that the element allows the reflection phase to be controlled through controlling active devices.

Several configurations of the electronically reconfigurable reflectarray elements have been proposed such as, microstrip patch loaded with varactor [1, 2], p-i-n diode or MEMS-based structures [3-5]. The electronically reconfigurable reflectarray elements can be designed using a single layer or multi-layers. The singlelayer configurations allow a simplicity in fabrication. However, the active devices arrange on the radiating surface, the DC-biasing network interferes to the radiation [1]. The elements based on aperture-coupled to delay line $[2,4-6]$ show considerable advantages. The interference problem caused by the DC-biasing network to the radiation can be overcome, since the active devices and its DC-biasing network are placed on the non-radiation surface. The element can provide the wideband characteristic thank to the capability of producing the true-time delay. Various kinds of active devices can be used with this kind of element to design electronically reconfigurable reflectarray antennas, such as Varactor [2], p-i-n diode [4], or MEMS [5, 6]. The integration of varactor to aperture-coupled elements can give a continuous phase adjustment. However, the drawback is the sensitivity of the reflection phase to DC control voltage. The digital approach with the use of switching devices such as p-i-n diodes or RF-MEMS can overcome the sensitivity problem. However, the number of phase states is the most important factor. Larger number of phase states leads to smaller phase error then smaller gain degradation [7]. For the aperturecoupled elements with a single delay line, for a number $N$ of switching devices, the number of phase state is limited to $N+1[5,6]$. Recently, a new aperture coupled reflectarray element based on the cut-ring patch coupled to the delay line has been investigated. This configuration has demonstrated a wide range of reflection phase with a very good linearity. The active devices such as $\mathrm{p}$-i-n diodes can be easily integrated into this element to implement electronically reconfigurable phase shifter [7]. Furthermore, the reflection phase can be controlled by modifying the length of two delay lines [8]. The configuration with two delay lines promises to allow a higher number of phase states compared to the case of one delay line for a same number of switching devices.

In this paper, we propose a 2-bit reflectarray element based on cut-ring patch coupled to two delay lines through an annular slot. This element is a development of the element studied in [8]. The element has been 
fabricated and measured to validate the design. Although it is still passive, we demonstrate the capability of providing 4 states of the reflection phase with a phase step of $90^{\circ}$, with only two switching devices.

\section{Element Configuration}

The 2-bit phase shifting reflectarray phase shifter is shown in Figure 1. The element is designed using multisubstrate layers. It consists of printed circuits, including fixed-sized cut-ring patch on the top plane, the annular slot on the coupling plane and the delay lines located on the bottom plane. The cut-ring patch acts as radiating element which is located on the top substrate. Two microstrip lines attached to circular patch are arranged on the bottom of the second substrate. The coupling ground separates the radiating element from the microstrip lines. The annular coupling slot allows signals to be coupled from the radiating element to the microstrip lines. The operation of the element can be understood that the incident wave after being received by the cut-ring patch is coupled to two delay lines through the annular slot. These coupled signals flow along the two lines. Because the two lines are open at the end, the coupled signals are reflected at the end of the line and they will be recouped to the cut-ring patch and be reradiated back in the free space. In order that the reflection phase can be changed independently with respect to the length of each line, a shorting pin is used to connect the inner circle of the annular slot to circular patch. This connection is to create the point zero of each line. The length of a line is determined by the distance from the zero point to the open end of that line. The circular patch attached to the delay line plays a role to tune impedance matching between the delay line and the cut-ring patch. The element operates with single polarization where the E-vector is along the y axis, as shown in Figure 1.

The RT/Duroid 5880 substrates with permittivity $\epsilon_{r}=2.2$ and dielectric loss tangent $\tan \delta=0.0009$ are used. The use of thick substrates is to achieve a wide bandwidth and also improve the linearity of the reflection phase curve. However, increasing the thickness of the substrates leads to an increase in the leakage wave. Therefore, the loss and the bandwidth need to be compromised.

Since the reflection phase of the reflectarray element is controlled by controlling the length of delay lines, switching devices such as p-i-n diodes or RF-MEMS can be used to be integrated to the reflectarray element in order to implement electronically configurable reflectarray antennas. In this case, switching devices are inserted between two segments of the delay lines. The change of reflection phase is controlled through the ON/OFF states of the switch. Indeed, when the switch is OFF, the electrical length of one delay line corresponds to the first line segment. If the switch is $\mathrm{ON}$, the electrical length corresponds to the sum of two segments. If one switch is used, a 1-bit phase shifter can be created to provide two phase states. The number of
Table I

Dimentions of the 2-bit Phase Shifting Element

\begin{tabular}{|l|l|l|}
\hline Cell size (mm) & $a=18$ & \\
\hline Cut-ring patch (mm) & $R_{\text {out }}=4.6, R_{\text {in }}=2.5$ & $t=1.0 \mathrm{~mm}$ \\
\hline Annular slot (mm) & $R_{\text {out }}^{\prime}=3.9, R_{\text {in }}^{\prime}=3.6$ & \\
\hline Circular patch (mm) & $R_{c}=1.5$ & \\
\hline Substrate 1 (mm) & $h_{1}=3.175$ & $\begin{array}{l}\epsilon_{r}=2.2, \\
\tan \delta=0.0009\end{array}$ \\
\hline Substrate 2 (mm) & $h_{2}=0.787$ & $\begin{array}{l}\epsilon_{r}=2.2, \\
\tan \delta=0.0009\end{array}$ \\
\hline Microstrip line (mm) & $\begin{array}{l}W=1 \\
L_{1}=3.2 ; L_{2}=6.2 \\
L_{3}=3.2 ; L_{4}=8.7\end{array}$ \\
\hline
\end{tabular}

phase states can be increased by increasing the number of switches.In this case, the length of the delay lines can be extended by bending, like the U-shape to provide more space for those switches. For the reflectarray element with one delay line, the number of phase states follows the rule: $N+1$ states for $N$ switching devices. Thanks to the capability of changing the phase through the length of two delay lines, the proposed element can provide a higher number of phase states compared to the element with one delay line for the same number of switches.

In this design, we optimized the element to provide four states of the phase with $90^{\circ}$ step at $11.8 \mathrm{GHz}$ through using only 2 switching devices. We note that this element is the first step towards the design of the 2-bit active reflectarray phase shifting element. It is still passive and there are no real active devices to be used. All switches are replaced by metallic strips. The ON state of the switch corresponds to the connection of two segments of the line through the strip. The OFF state is when the strip is removed. The optimized parameters of 2-bit phase shifting element are reported in Table I.

\section{Simulation Results}

Once the design principle is defined, the parameters are optimized using commercial ANSYS HFSS software. First, the cut-ring patch is designed to operate at $11.8 \mathrm{GHz}$, the delay line is designed to have a characteristic impedance of $50 \Omega$. Then, the whole structure is optimized to achieve the low loss and good linearity of the phase curve. Since a reflectarray antenna consists of many reflecting elements which are placed in an array, the reflectarray element must be analyzed in an array environment to take into account the mutual coupling of the neighboring elements. The waveguide simulator approach is very efficient to analyze the element. With this approach, the infinite periodic array model can be reduced to only one unit cell.

Figure 2 shows the simulation setup to analyze the element using HFSS simulation software. The simulated cell is inserted into a waveguide with appropriate periodic boundary conditions. Here, the right and left walls of the waveguide are magnetic field walls and the top and bottom surfaces of the waveguide are electric conducting walls. 


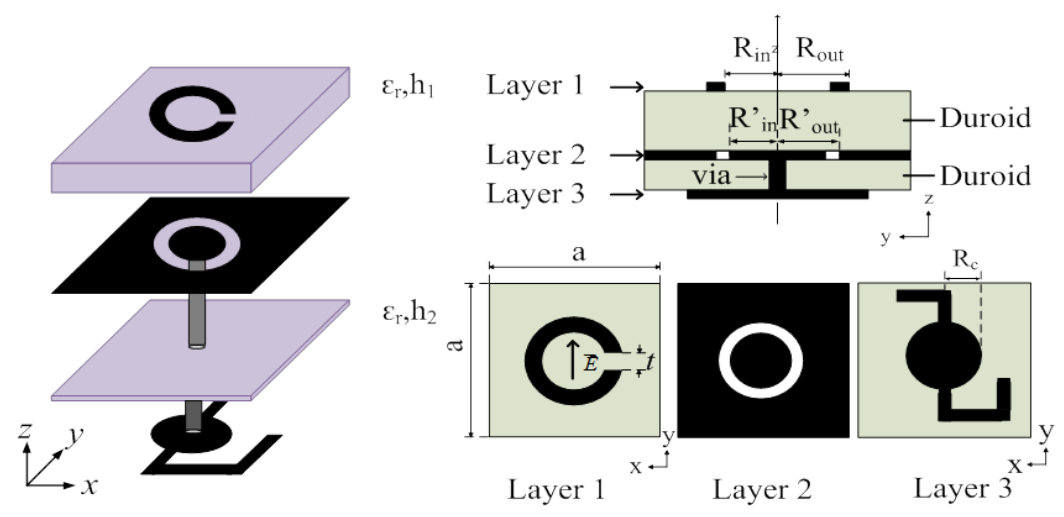

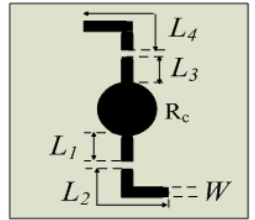

a)

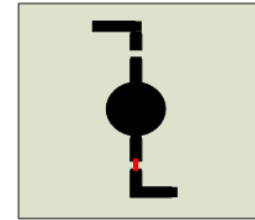

b)

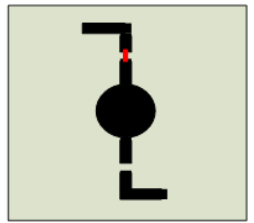

c)

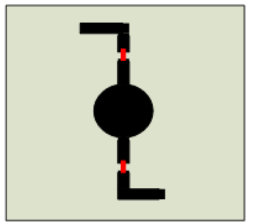

d)

a) State $1(00)$; b) State $2(01)$; c) State 3(10); d) State 4 (11)

Figure 1. Configuration of the element.

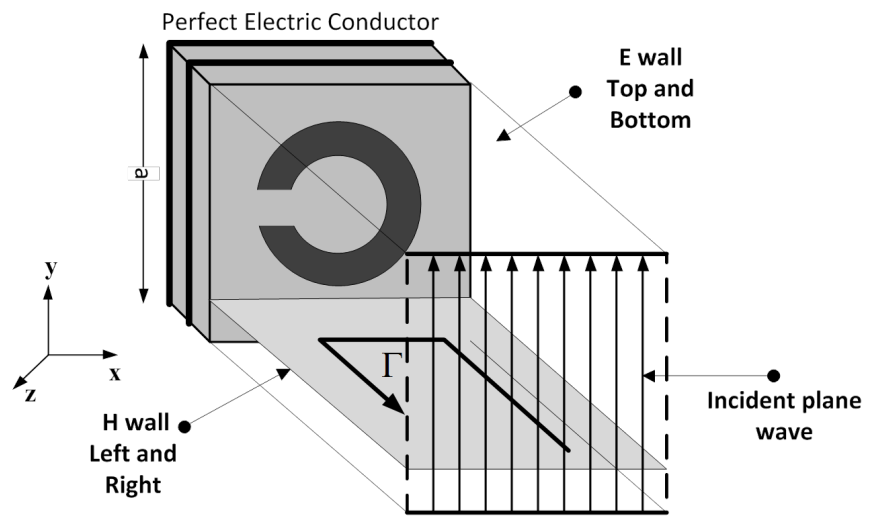

Figure 2. Simulation setup using waveguide simulator.

In analyzing a refectarray element, the phase and amplitude of reflected wave are the most important parameters to evaluate the performance of the element. Four combinations of segments of the two lines corresponding to four states $(00,01,10,11)$ have been simulated. The 00 state corresponds to the case when all metallic strips are removed. The 11 state happens when two strips are used to connect two segments of two delay lines. The 01,10 states happen when two segments of one delay line are connected through a strip. Figure 3 shows the simulated reflection phase and magnitude for a normal incident wave. The reference plane to analyze the reflected wave is the surface of the top substrate layer. It can be observed that four consecutive phase states are circa $90^{\circ}$ different at $11.8 \mathrm{GHz}$. Due to the nonlinearity of the phase curve, the difference of $90^{\circ}$ cannot be maintained at other frequencies. Large difference of phase between states occurs at frequencies far from $11.8 \mathrm{GHz}$. In order to evaluate the performance of the proposed element, the phase deviation $\sigma$ and the bit number $N_{\text {bit }}$ are examined. According to [9], the deviation $\sigma$ and the bit number $N_{\text {bit }}$ are shown in Equations (1) and (2).

$$
\begin{gathered}
\sigma=\sqrt{\frac{\sum_{i=1}^{4}\left(\triangle \theta_{i}\right)^{3}}{12 * 360},} \\
N_{\text {bit }}=\frac{\ln \left(\frac{360}{\sqrt{12} * \sigma}\right)}{\ln 2},
\end{gathered}
$$

where $\theta_{i}$ is the phase difference between the two adjacent states.

For a 2-bit phase element, the ideal value of the phase deviation $\sigma$ is $26^{\circ}$ and the bit number $N_{\text {bit }}$ is 2 . From the Figure 3 , the phase deviation $\sigma$ is $26.8^{\circ}$ at the working frequency of $11.8 \mathrm{GHz}$. It corresponds to a bit number of nearly 2. At $11.4 \mathrm{GHz}$, the deviation $\sigma$ is $33.0^{\circ}\left(N_{\text {bit }}\right.$ $=1.7$ ) and at $12 \mathrm{GHz}$, the phase deviation $\sigma$ is $29.3^{\circ}$ $\left(N_{\text {bit }}=1.7\right)$. The bandwidth for a deviation lower than $33.0^{\circ}\left(N_{\text {bit }}=1.7\right)$ is over $5 \%$. The magnitude losses are less than $0.8 \mathrm{~dB}$ for the band from $1.4 \mathrm{GHz}$ to $12 \mathrm{GHz}$.

\section{Validation Results}

In practice, a common method to validate the performance of a reflectarray element in terms of reflection phase and magnitude is to use the waveguide simulator (WGS). With this approach, fabricated elements are inserted into a waveguide. The reflection phase and reflection magnitude will be determined by measuring the reflection coefficient (S11). Several prototypes containing 2 identical cells have been fabricated. The WR-90 standard waveguide was used as a waveguide simulator since the working frequency band is from $8.2-12.4 \mathrm{GHz}$. In order to insert the prototype into the waveguide, an open-end tapered from the WR-90 


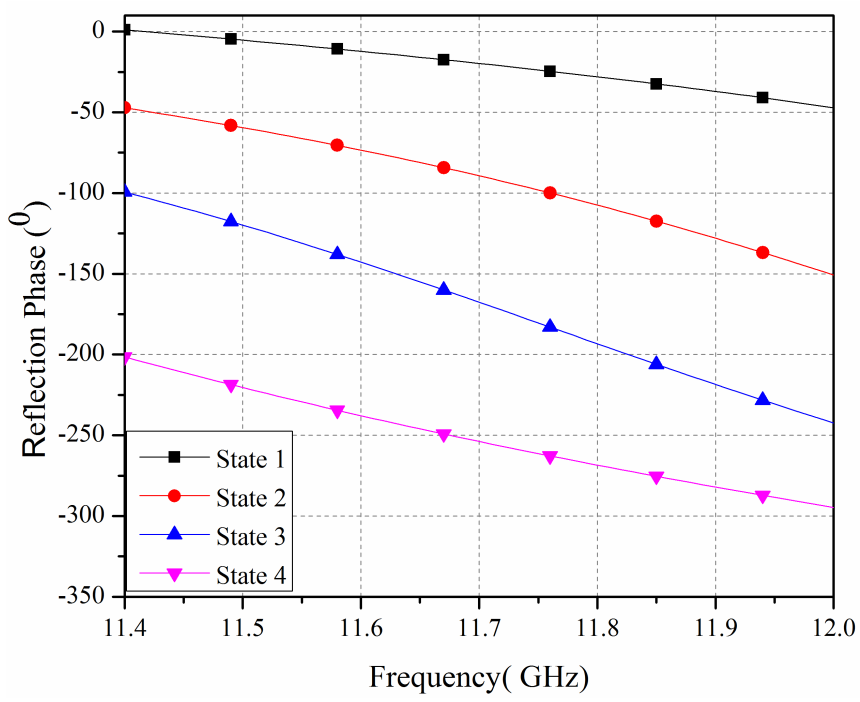

(a) Phase response.

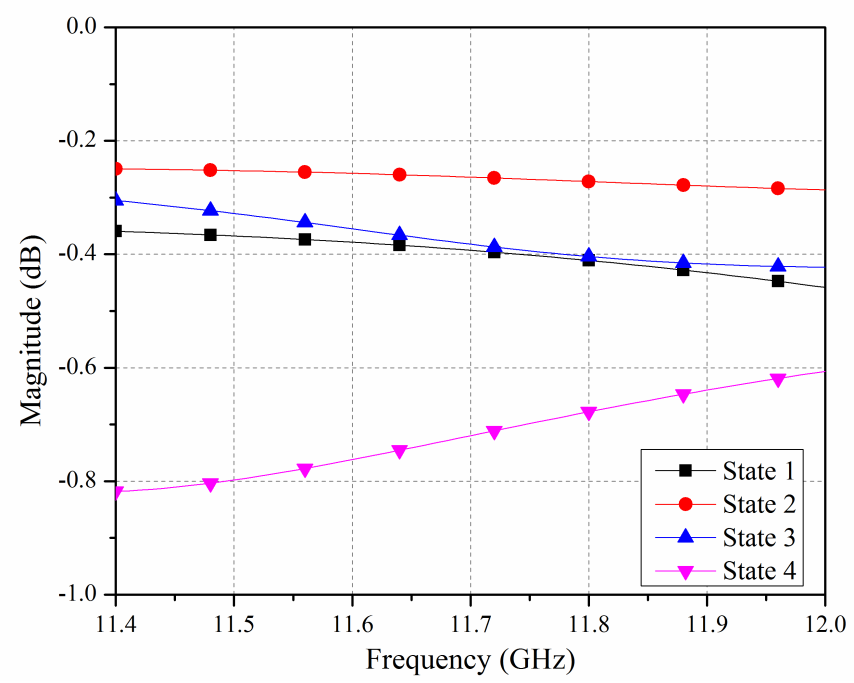

(b) Amplitude response.

Figure 3. Simulated magnitude and phase of reflected wave for four states.

standard waveguide was implemented. The surface at the open-end of the tapered waveguide is $18 \times 36 \mathrm{~mm}$ which corresponds to the surface of the prototype. Measurement of the phase and magnitude of the reflected wave was carried out by Network Analyzer by measuring the reflection coefficient (S11) at the excitation port. The reference plane is at $170 \mathrm{~mm}$ from the top layer of element. This is the length of the openend tapered waveguide from the open-end to the probe. Each delay line of the cell is divided into 2 segments which are separated by a gap of $0.3 \mathrm{~mm}$. Depending on the state, the metallic strips were used to connect two segments of one or two delay lines in order to produce 4 states.

The simulated and the measured results are shown in Figure 6. There is a good agreement between simulation and measurement in terms of reflection phase. Measured results show that the phases of adjacent states are nearly separated by $90^{\circ}$ at $11.8 \mathrm{GHz}$ and they maintain a good distance in the band $11.4-12.0 \mathrm{GHz}$. The phase deviation $\sigma$ is $29.1^{\circ}\left(N_{\text {bit }}=1.8\right)$ at $11.4 \mathrm{GHz}$ and

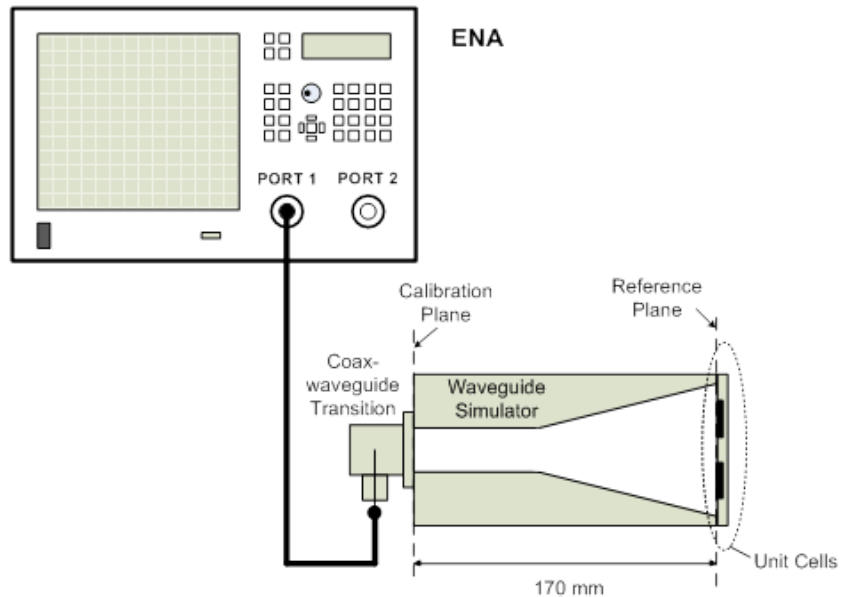

Figure 4. Measurement system.

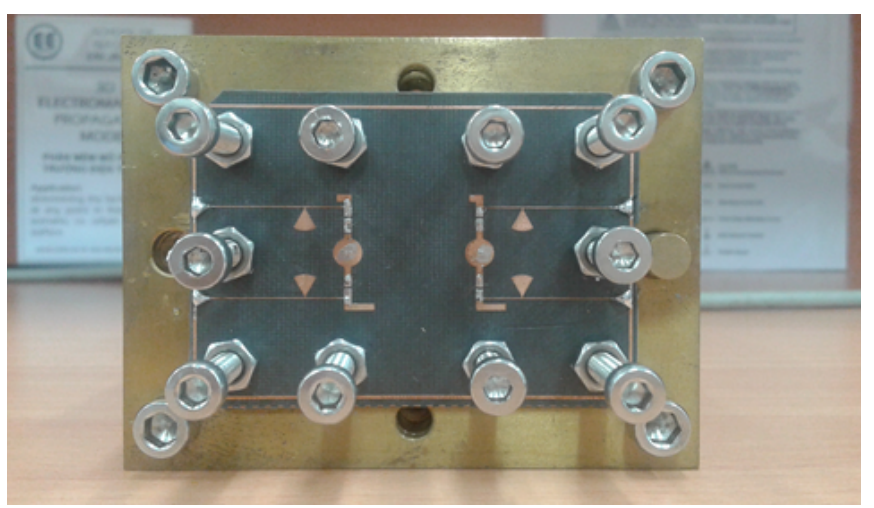

Figure 5. Photo of a prototype.

the phase deviation is $31.7^{\circ}\left(N_{\text {bit }}=1.7\right)$ at $12 \mathrm{GHz}$. The magnitude losses are slightly higher than those of simulations. The average losses of around $1.2 \mathrm{~dB}$ are obtained in the band $11.4-12.0 \mathrm{GHz}$. The difference between simulation and measurement results may be due to the imperfect alignment among the substrates, manufacturing tolerances.

\section{Conclusion}

The proposed two-bit reflectarray element has been validated. Four states of the reflection phase are obtained through modifying the length of two delay line. The measurement has validated the capability of providing four states with two switches. This is the main advantage of the proposed configuration compared to the element based on aperture-coupled to one delay line. Actually, the element is still passive. For the future work, the real active devices will be inserted to the elements in order to validate the design.

\section{AcKnowledgment}

This research is funded by Vietnam National Foundation for Science and Technology Development (NAFOSTED) under grant number 102.99-2012.38. 


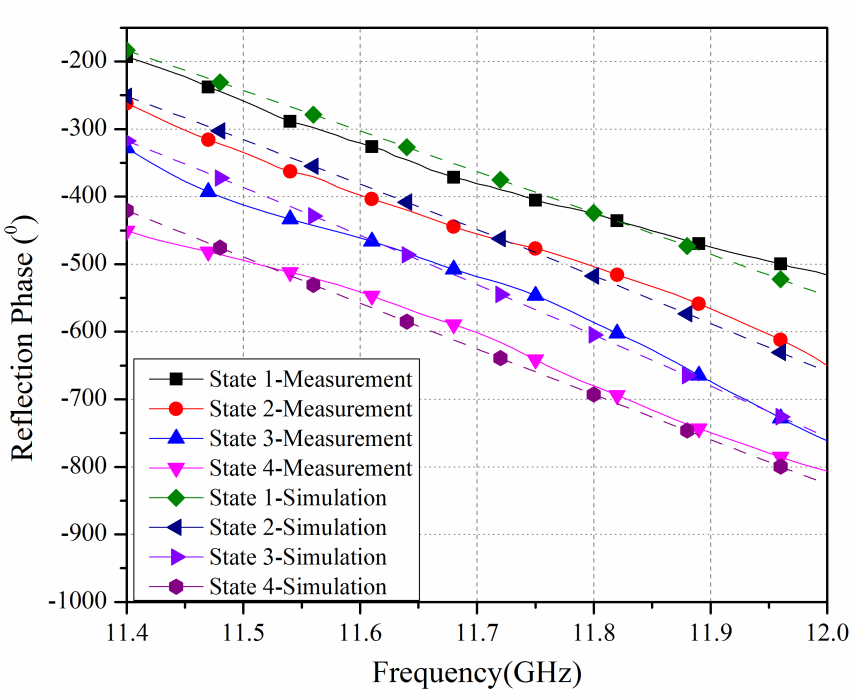

(a) Phase response.

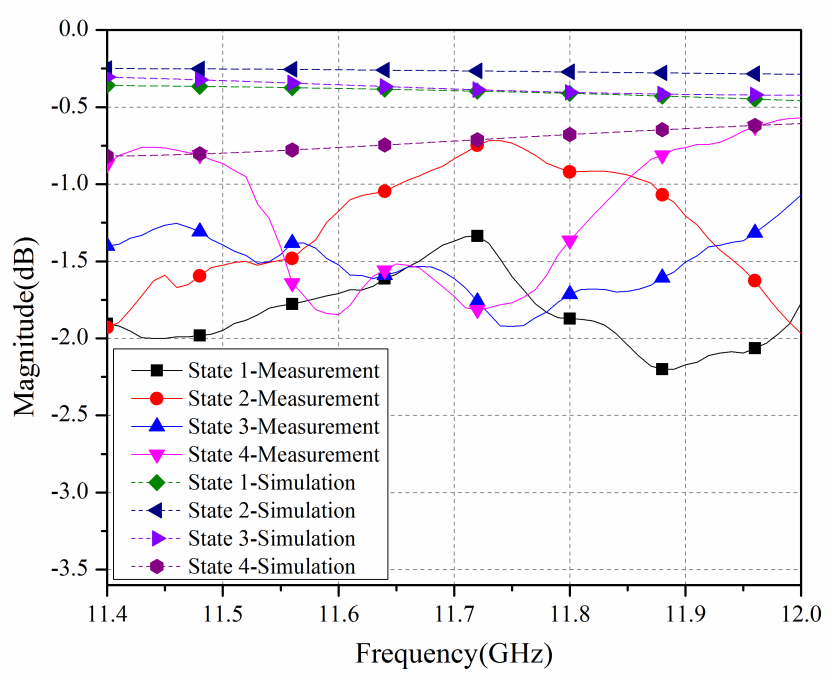

(b) Amplitude response.

Figure 6. Measured magnitude and phase of reflected wave for four states.

\section{REFERENCES}

[1] O. Vendik and M. Parnes, "A phase shifter with one tunable component for a reflectarray antenna," IEEE Antennas and Propagation Magazine, vol. 50, no. 4, pp. 53-65, Aug. 2008.

[2] M. Riel and J. Laurin, "Design of an Electronically Beam Scanning Reflectarray Using Aperture-Coupled Elements," IEEE Transactions on Antennas and Propagation, vol. 55, no. 5, pp. 1260-1266, May 2007.

[3] H. Kamoda, T. Iwasaki, J. Tsumochi, T. Kuki, and O. Hashimoto, "60-GHz Electronically Reconfigurable Large Reflectarray Using Single-Bit Phase Shifters," IEEE Transactions on Antennas and Propagation, vol. 59, no. 7, pp. 2524-2531, Jul. 2011.

[4] E. Carrasco, M. Barba, and J. Encinar, "X-Band Reflectarray Antenna With Switching-Beam Using PIN Diodes and Gathered Elements," IEEE Transactions on Antennas and Propagation, vol. 60, no. 12, pp. 5700-5708, Dec. 2012.

[5] O. Bayraktar, O. Civi, and T. Akin, "Beam Switching Reflectarray Monolithically Integrated With RF MEMS Switches," IEEE Transactions on Antennas and Propagation, vol. 60, no. 2, pp. 854-862, Feb. 2012.

[6] E. Carrasco, M. Barba, B. Reig, C. Dieppedale, and J. Encinar, "Characterization of a Reflectarray Gathered Element With Electronic Control Using Ohmic RF MEMS and Patches Aperture-Coupled to a Delay Line," IEEE Transactions on Antennas and Propagation, vol. 60, no. 9, pp. 41904201, Sep. 2012.

[7] B. D. Nguyen, K. T. Pham, V.-S. Tran, L. Mai, N. Yonemoto, A. Kohmura, and S. Futatsumori, "Electronically tunable reflectarray element based on C-patch coupled to delay line," Electronics Letters, vol. 50, no. 16, pp. 1114-1116, Jul. 2014.

[8] B. Nguyen, K. Pham, V.-S. Tran, L. Mai, and N. Yonemoto, "Reflectarray Element Using Cut-Ring Patch Coupled to Delay Line," IEEE Antennas and Wireless Propagation Letters, vol. 14, pp. 571-574, 2015.

[9] R. Pereira, R. Gillard, R. Sauleau, P. Potier, T. Dousset, and X. Delestre, "Dual Linearly-Polarized Unit-Cells With Nearly 2-Bit Resolution For Reflectarray Applications In X-Band," IEEE Transactions on Antennas and Propagation, vol. 60, no. 12, pp. 6042-6048, Dec. 2012. 